\title{
Testing Graphene Films Produced Through Electrochemical Exfoliation Using Sulfate Electrolytes
}

\author{
Samuel Blankenship 1 , Stefanie Davis ${ }^{\#}$ and Mangilal Agarwal ${ }^{\#}$ \\ ${ }^{1}$ Perry Meridian High School, Indianapolis, IN, USA \\ \#Mentor
}

\section{$\underline{\text { ABSTRACT }}$}

Graphene is one of the most commonly researched materials in nanoscience and finding a cheap and efficient method to manufacture it is highly desirable because of its incredible properties. Electrochemical exfoliation involves splitting graphite into graphene by soaking the foil in an electrolyte solution and then providing an electric current. This paper evaluates the extent to which the sulphate electrolyte used in the electrochemical exfoliation process affects the electrical resistance of films created using flakes generated from the reaction. Using the method and conducting an ANOVA test with Tukey HSD Post-Hoc test on the resultant data provides significant and varied results when concerning the electrolyte variety. This implies that changing the quality and speed of the electrolyte reaction has a definitive effect on the resistance of composite films created out of graphene flakes produced from the reaction.

\section{Introduction}

Graphene is one of the most commonly investigated materials in the current age of materials science and engineering. It consists of sheets of hexagonally oriented carbon atoms kept loosely together by Van der Waals forces, which exhibit drastically different types of behavior depending on the number of layers stacked together. ${ }^{2}$ Graphene monolayers, which consist of single, separated sheets of graphene, are generally the most publicized forms of graphitic materials due to their incredible conductive and resilient traits. They have a wide variety of applications ranging from electronics to protective equipment but are difficult to manufacture due to the atomic level of precision required during production.

Graphene fabrication methods are categorized into bottom-up approaches and top-down approaches. Bottomup approaches allow for tight control of the end product at the cost of smaller and more expensive yields, while topdown approaches produce more unpredictable results in much greater quantities. ${ }^{3}$ This study focused on the top-down approach of electrochemical exfoliation, which uses one or more electrolytes in a dielectric solvent to chemically shear graphite into flakes. The process pulls ions from the electrolyte into the gaps between the graphite sheets, and then uses an electric current to cause the ions to expand the surrounding area and push the sheets away from each other. This method was originally used with acid and oxidized graphite to create graphene oxide, another form of graphene primarily used in the biomedical industry, and as a stepping stone to create graphene. However, this method created low-quality graphene sheets and was therefore labeled as obsolete when more advanced methods were developed. ${ }^{4}$ The method would essentially remain untouched until scientists began to find new methods to separate fewlayer graphene from monolayer graphene.

Recent revisitations to the concept involving the direct exfoliation of graphite into graphene have led to a great deal of interesting outcomes, with some methods promising monolayer graphene yields of over $60 \%$ compared to the graphite base. ${ }^{5}$ Once purified through weight-based separation techniques, the graphene sheets can be used for a plethora of applications, which range from being condensed into sheets for electronic use to being mixed into epoxies for aeronautical purposes. 
Although this revisitation is promising, no study has previously been conducted that directly correlates the electrical resistances of graphene films produced using different electrolytes in an electrochemical exfoliation reaction and the electrolyte choice itself. Completion of such a study would allow for future experiments to easily determine the expected results that occur from using a certain sulfate electrolyte in film creation, and therefore be able to use the electrolyte that best matches the desired level of conductivity. This could potentially allow for further work to be done in the area of film characterization based on electrolyte type used in the reaction, continuing to deepen the scientific community's understanding of the qualities of graphene films built from graphene flakes.

The study conducted in the paper focused on varying the electrolytes suspended in the electrochemical exfoliation medium and determining the effects of these changes on the resistance of films created using the resulting graphene flakes in order to vary the effective yield of graphene monolayers in the electrochemical exfoliation reaction. The following section reviews the findings of other members in the community surrounding electrochemical exfoliation and describes the gap in research this paper determines to answer.

\section{Literature Review}

Electrochemical exfoliation has a wide number of variables involved in determining the final product's quality and quantity, which has caused a great deal of research to be done by manipulating these factors. The variables that are most often altered during experimentation include the electrolyte used to exfoliate the graphene and the amount of energy used during the reaction, both of which drastically affect the outcome of the reaction.

When the process was initially developed, acid was the most common electrolyte used in the reaction, and the results were comparatively low. A study conducted by $\mathrm{C}$. Su et al. used this method and had a final monolayer graphene yield of roughly $5-8 \% .^{6}$ This amount is incredibly low, as it means $92-95 \%$ of the exfoliated graphite is either too small to be usable or stacked in multiple layers due to an incomplete shearing process, but the process was rapid compared to bottom-up methods like carbon vapor deposition.

The main competing method to electrochemical exfoliation is carbon vapor deposition because it can produce high-quality sheets of graphene in a continuous and scalable process. It primarily involves facilitating the conditions necessary for carbon to condense onto a substrate, creating an almost perfect sheet of graphene made from millions of small micron-wide crystals. The near-perfect quality of the sheets has attracted a great deal of attention from researchers, and a variety of comparatively large-scale experiments have been conducted using these sheets. However, carbon vapor deposition suffers from expensive fabrication procedures, traditionally requiring a pure gas medium with temperatures in excess of $1000{ }^{\circ} \mathrm{C}$ for crystal growth. ${ }^{7}$ Just like electrochemical exfoliation, though, it has not been exempted from major advancements. $\mathrm{Z}$. Li et al. have made the carbon vapor deposition considerably more viable for industrial production by using specific gas mediums and substrates to lower the temperature required to a far more reasonable $300{ }^{\circ} \mathrm{C} .{ }^{7}$ This has made it more financially competitive compared to electrochemical exfoliation, with a far more reliable film yield. However, the equipment required to produce graphene through carbon vapor deposition is still expensive, limiting the method's use to high-budget projects.

Because of the promising speed and cost-effectiveness of electrochemical exfoliation, studies conducted in more recent years attempted to improve the final products' qualities. The following year, D. Wei et al. discovered that adding lithium salts to the exfoliation process improved the efficiency of the reaction and improved the end results by a considerable degree. ${ }^{8}$ This development sparked a huge amount of future research because it suggested that in addition to acidic materials like sulphuric acid and $\mathrm{BMIMBF}_{4}$, other ionic substances can also be used as viable electrolytes for the electrochemical exfoliation reaction. Tripathi et al. continued along this train of thought by experimenting with more electrolytes, finding additional improvements by using $\mathrm{KOH}$, potassium hydroxide, as an electrolyte. ${ }^{9}$ Although alkaline electrolytes have allowed for possible improvements in graphene flake quality, the danger of utilizing highly alkaline chemicals prevented this paper from exploring in this area. Electrolytes are one of the primary methods to improve graphene flake quality during electrochemical exfoliation, but there has been a considerable amount of research done in a second critical field. 
The second major area of variable manipulation involves changing the type of graphitic anode used in the reaction, since the orientation of exposed graphite during the reaction has shown to be impactful on the final product's quality. The two main shapes of anode used are usually either sheets of a thin foil or rods composed entirely out of graphitic material, but other studies have attempted to alter this further to see which results are the most beneficial. J. Liu et al. experimented with using a cylindrical rod of graphite inside of a test tube instead of two parallel sheets of foil and concluded that a less large surface area leads to a more complete and efficient exfoliation. ${ }^{10}$ This development could lead to a complete reimagining of how the electrochemical exfoliation reaction vessel is designed, both for experimental and industrial purposes, and $\mathrm{H}$. Wang et al. decided to further pursue this area just six months later. Their study involved exfoliating graphite foil pieces covered with different levels of paraffin wax, and it concluded that increasing the confinement of the graphite anode can lead to a significant improvement in graphene flake quality. ${ }^{5}$ Although they determined a correlation between confinement and graphene flake quality following electrochemical exfoliation, an ideal confinement method has not been determined. Therefore, this study refrained from using confined graphite to keep results consistent with most other studies.

Graphene films are one of the premiere end products of the small sheets of graphene created from electrochemical exfoliation. They have a variety of uses in the electronics field, and mass-production of them would revolutionize the electronics industry due to graphene's incredibly low electric resistance. H. Wang et al. described the process required to transform graphene flakes into films, and the method they used to test it. ${ }^{5}$ After graphene exfoliation, the graphene was prepared in a dispersion and filtered through a vacuum filter with a fine membrane filter. ${ }^{5}$ After this, the flakes were washed repeatedly, taken off the filter by floating the flakes in water, and deposited on a quartz substrate..$^{5}$ These methods formed the basis for the study's own methodology, although some aspects were removed to regulate variables.

\section{Methods}

This study centered around the electrical resistance testing of graphene films. The graphene was produced using electrochemical exfoliation, deposited onto a fused quartz substrate, and then tested using a multimeter to find its resistance.

Electrochemical exfoliation is a process that transforms graphite into few-layered graphene flakes using an electrolyte solution and electricity. It functions by soaking a form of graphite in a solution of distilled water and one or more electrolytes, and then applying an electric current. The ions present in the electrolyte pull themselves into the gaps between the graphite sheets, and when the current is applied, they become excited and expand, pushing the layers apart in flakes.

The form of graphite used in this study for exfoliation was a $0.1 \mathrm{~mm}$ thick foil. The foil was cut into $30 \mathrm{~mm}$ by $100 \mathrm{~mm}$ pieces to best fill the reaction chamber, connected to the reaction chamber by electrical tape to keep it stable during the reaction, and partially inserted into the reaction chamber after the solution was added. The reaction chamber consisted of a large, clean glass beaker that was filled with the electrolyte solution, with foil attached to opposite ends of it. Figure 1a depicts the experimental setup as used for this experiment. This study used a variety of electrolytes in different molarities and variations in order to determine the effectiveness of the respective electrolyte solutions in making successfully conductive graphite films. The electrolytes used in a solution of distilled water include ammonium sulfate $\left(\left(\mathrm{NH}_{4}\right)_{2} \mathrm{SO}_{4}\right)$, potassium sulfate $\left(\mathrm{K}_{2} \mathrm{SO}_{4}\right)$, and sodium sulfate $\left(\mathrm{Na}_{2} \mathrm{SO}_{4}\right)$. The solution molarities were $0.1 \mathrm{M}$ and $0.2 \mathrm{M}$, for each of the three electrolytes. Power was provided through the use of two electrodes, one placed on each of the graphite foil pieces. The voltage exerted was a constant $10 \mathrm{~V}$ of direct current during all reactions. Figure $1 \mathrm{~b}$ illustrates the reaction in progress, and Figure 1c shows the flakes produced after exfoliation. The process creates hydrogen and oxygen gas, which were siphoned off by the fume hood before they could build up and produce explosive reactions. 


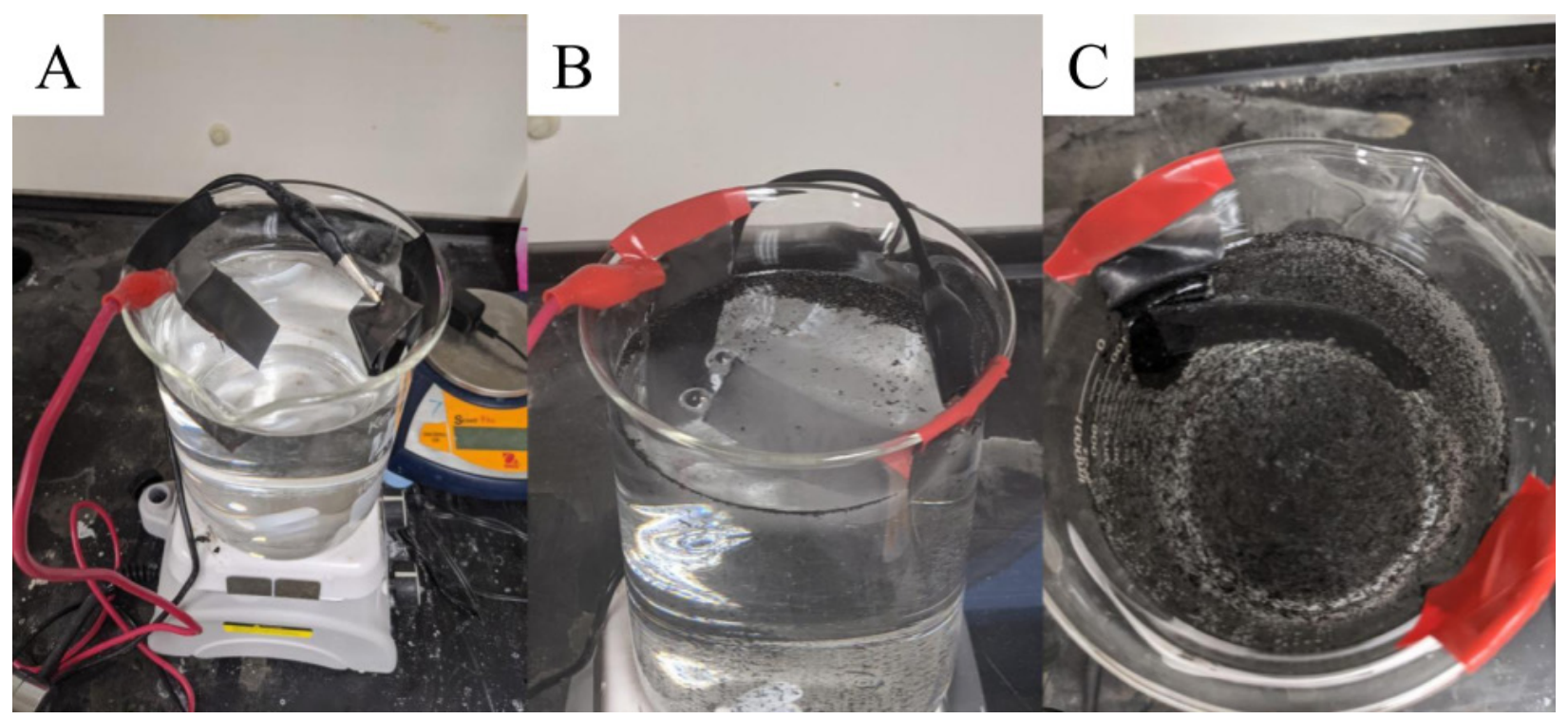

Figure 1. Images from various stages in the electrochemical exfoliation reaction. Figure 1a demonstrates the equipment and materials used for electrochemical exfoliation. Figure $1 \mathrm{~b}$ illustrates the reaction itself. Figure 1c documents the completed reaction.

\section{Materials}

The $0.1 \mathrm{~mm}$ graphite foil was purchased from MinGraph via Amazon and serves as the graphite source for the reaction. Foil was chosen because of its ease of access and high surface area compared to other forms like graphite rods, and $0.1 \mathrm{~mm}$ provides an economical mix between high surface area and stability during experimentation. The ammonium sulfate $\left(\left(\mathrm{NH}_{4}\right)_{2} \mathrm{SO}_{4}\right)$ and potassium sulfate $\left(\mathrm{K}_{2} \mathrm{SO}_{4}\right)$ were purchased from Alpha Chemicals via Amazon, and the sodium sulfate $\left(\mathrm{Na}_{2} \mathrm{SO}_{4}\right)$ was purchased from Duda Energy via Amazon. Each served as an electrolyte used in the reaction. Other common electrolytes used for electrochemical exfoliation, like sodium hydroxide and sulfuric acid, are highly caustic and reactive. This study will use these chemicals instead because they still produce viable graphene at a low hazard to the chemist. The nylon membrane filters were purchased from Foxx Life Sciences via Amazon and were chosen because of their chemical compatibility and small pore size. The $50.8 \mathrm{~mm}$ diameter fused quartz substrates (3.175 mm thickness) were purchased from Pelco via Ted Pella and were used to collect the graphene films because of their high-quality and dielectric nature.

\section{Preparation of Films}

After the reaction has occurred and the graphene has been exfoliated, power was cut, the solution was stirred, and the chamber was then left to settle overnight. Once this time passed, the graphene separated itself by weight and therefore layer count. Monolayer graphene concentrated itself near the top, while multilayer graphene dropped to the bottom. The top layer was poured off onto a nylon membrane filter-covered Büchner funnel, which was placed over a clean vacuum flask. Then, the graphene was washed with distilled water to remove excess electrolytes and other impurities. The resulting graphene flakes were stored in vials and placed into an ultrasound cleaning device. After being sonicated for 1 hour, the solution was again left to settle overnight and stored for later use. Figure 2a depicts the vials of solution shortly before being left to settle.

Before film fabrication, the vials were sonicated for a further 30 minutes and dumped along with additional distilled water into a clean vacuum filter lined with another nylon membrane. Figure $2 \mathrm{~b}$ illustrates the filtering process. 
After the filtering process was complete, the filter was removed and carefully dropped into a container filled with distilled water. The filter was then separated and fell to the bottom, leaving multiple small films of graphene on top of the water as shown in Figure 2c. Then, a fused quartz substrate was used to harvest the films and prepare them for testing. Figure $2 \mathrm{~d}$ shows one of the larger film batches shortly after collection, made using the $0.2 \mathrm{M}$ sodium sulfate solution.

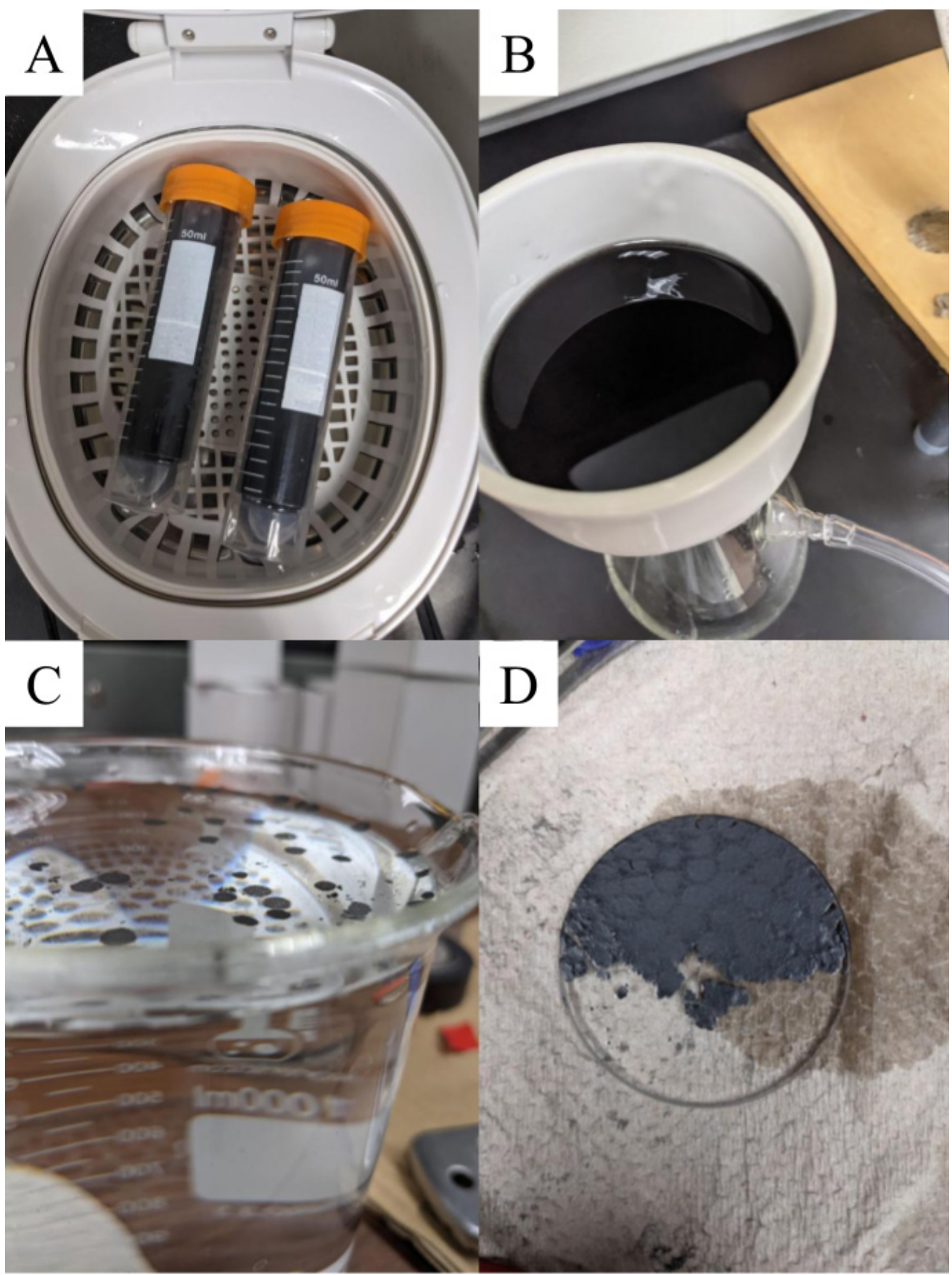

Figure 2. Images from the film preparation process. Figure 2a documents the vials after 1 hour of sonication. Figure $2 \mathrm{~b}$ depicts the vacuum filtering process. Figure $2 \mathrm{c}$ is a focused image of the floating graphene films shortly before they are collected on a substrate. 


\section{Film Testing}

Testing consisted of utilizing a multimeter on the surface of the film to check for electrical resistance in different areas. The two ends of the multimeter were placed directly across from each other, on concentric ends of the circular film. Measurements were taken in $1 \mathrm{~mm}$ increments, increasing the distance between the two pins by $1 \mathrm{~mm}$ each time and testing each film at multiple different angles to ensure consistency. After collecting the measurements from a given film cluster, the substrate was cleaned and the experiment was repeated, using each electrolyte combination twice to ensure consistency. Between each experiment, the filters and foil were replaced, the electrolyte solution disposed of properly, and all containers were washed and dried thoroughly. The experiment was only repeated twice for each electrolyte combination due to a combination of time and budget constraints. In addition, a four-point probe system could be utilized to gain far more precise resistance measurements, but budget constraints again limited the research to using a traditional multimeter.

The methods used in the paper match the goal previously stated because they directly serve to answer the question proposed by the study through scientific research. The resistance of graphene films made using different molarities and varieties of electrolytes was measured and analyzed to directly verify the correlation between electrolyte type and the resistance of graphene.

The method originated from a synthesis of different methods used in this field of research, combining graphene film-making procedures with electrolytes used in other experiments. ${ }^{35}$ Other studies have already proposed there is a considerable difference in graphene flake quality based on the type and molarity of certain electrolytes, and this study expanded on the concept by verifying the correlation between electrolyte type and graphene film resistance.

${ }^{3}$ When attempting to apply these methods in the electronics industry, it is incredibly important to know whether using a different electrolyte solution will have a direct and substantial impact on the final resistance of electronics made using the flakes. This research attempts to evaluate whether there is a correlation, and if so, how strong it is.

\section{Safety}

This method used three potentially hazardous materials and was verified by both Perry Meridian High School and an IRB in order to ensure proper safety was undertaken.

Ammonium sulfate, potassium sulfate, and sodium sulfate all share similar dangers, which include minor skin irritation on skin contact, potential eye damage on eye contact, and poisonous reactions if inhaled or ingested. They also become unstable when exposed to high temperatures, so they were stored at room temperature. A fume hood, thick latex gloves, chemical-resistant goggles, a respirator, long-sleeved clothing, and closed-toe shoes covered the other risks, and caution was taken when handling the chemicals. Disposal of the electrolyte solutions first involved sieving out the larger pieces of graphite left in the solution. Then, the electrolyte solutions were simply poured down a drain as it is safe to do so. The disposable containers used for graphene suspension storage, the remaining reacted foil, the filters used for washing, and leftover electrolytes were disposed of in a waste disposal facility following the experiment.

Any spillage of electrolyte-based material was cleaned up immediately and safely, taking care not to generate dust and avoid soil/water contamination.

\section{Results}

After obtaining the electrical resistance measurements and recording them on a spreadsheet, the data was coded to account for the distance at which the measurements were taken, and the type of solution used to make the films that 
were tested. Then, the results were imported into the DataClassroom software for graphing and testing. Figure 3 depicts the resulting graph, with added jitter for illustration purposes. Each dot is a measurement of a film's resistance taken from a distance that corresponds to the color of the dot. The $\mathrm{x}$-axis represents the type of solution used when making the tested film, with each solution type used being coded as a number from one to six. The y-axis is the sole measured dependent variable of the experiment, with film resistances ranging from $45 \boldsymbol{\Omega}$ to $1.79 \mathrm{k} \boldsymbol{\Omega}$.

A scatter chart was chosen for this form of data representation since it was the only medium capable of properly displaying hundreds of data points on multiple axes. Because further tests require all data to be coded, it was done preemptively.

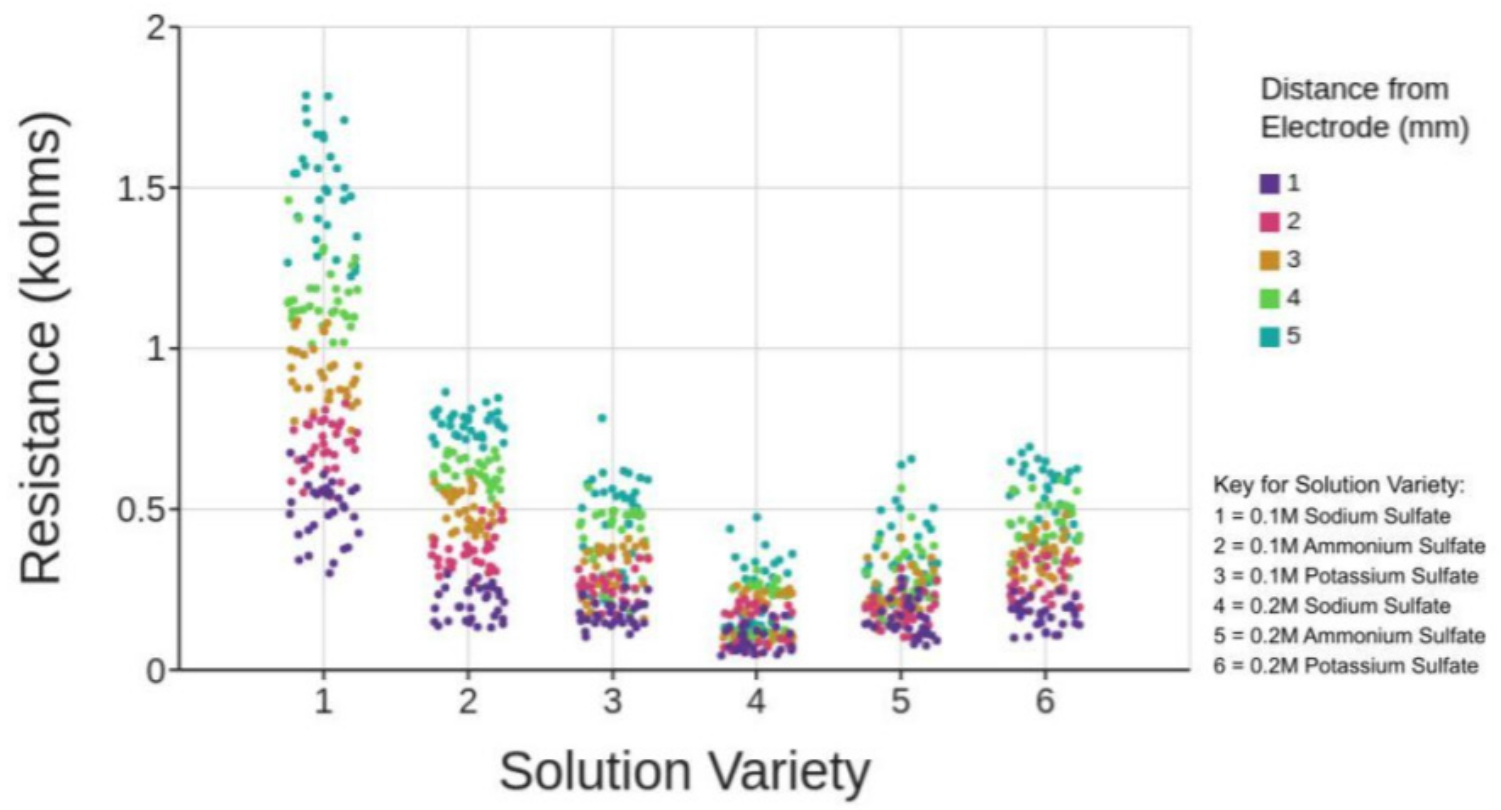

Figure 3. A graph of the solution varieties and resistances of the respective graphene films, with colors to depict the results at different electrode distances.

Once the data was graphed, a 1-way ANOVA test was conducted to determine whether the relationship between the solution variety and the film resistance was significant. This test was used because it was the only readily available statistics test capable of finding the relationship between multiple $\mathrm{x}$-axis groups. Because the figure was not being tested, the distance from electrodes was excluded from statistical analysis. Table 1 documents the results from the test, which conclude that there is a $>99 \%$ chance that the null hypothesis was rejected. A rejected null hypothesis means there is a significant difference between the electrolytes, implying that changing the electrolyte will change the resistance of the graphene film.

Table 1. A table that depicts the 1-way ANOVA test results.

\begin{tabular}{|l|l|l|l|}
\hline Effect & $\begin{array}{l}\text { Degrees of Freedom } \\
\text { (df) }\end{array}$ & $\begin{array}{l}\text { F-Statistic } \\
\text { (MS/MS Residual) }\end{array}$ & P-Value \\
\hline Solution Variety & 5 & 320 & $<0.01$ \\
\hline
\end{tabular}


A standard ANOVA test does not describe which groups are statistically different from each other, which should be considered in this situation. To remedy this, the Tukey HSD post-hoc test was selected and applied via the astatsa.com website program to determine which groups were statistically different from each other. Table 2 depicts the results of the Tukey HSD test and finds that every combination except for $\mathrm{C}$ and $\mathrm{F}$ have a statistical difference compared to each other. This means that in every case except for $\mathrm{C}$ and $\mathrm{F}$, exchanging the electrolyte used in the reaction will have an effect on the outcome of the film resistance.

Table 2. A table that depicts the Tukey HSD post-hoc test results.

\begin{tabular}{|l|l|l|l|}
\hline Treatments Pair & Tukey HSD Q Statistic & Tukey HSD P-Value & Tukey HSD Interference \\
\hline A vs B & 29.671 & 0.0010053 & $\mathrm{p}<0.01$ \\
\hline A vs C & 40.1131 & 0.0010053 & $\mathrm{p}<0.01$ \\
\hline A vs D & 50.4494 & 0.0010053 & $\mathrm{p}<0.01$ \\
\hline A vs E & 44.8246 & 0.0010053 & $\mathrm{p}<0.01$ \\
\hline A vs F & 37.7436 & 0.0010053 & $\mathrm{p}<0.01$ \\
\hline B vs C & 10.442 & 0.0010053 & $\mathrm{p}<0.01$ \\
\hline B vs D & 20.7783 & 0.0010053 & $\mathrm{p}<0.01$ \\
\hline B vs E & 15.1536 & 0.0010053 & $\mathrm{p}<0.01$ \\
\hline B vs F & 8.0725 & 0.0010053 & $\mathrm{p}<0.01$ \\
\hline C vs D & 10.3363 & 0.0010053 & $\mathrm{p}<0.01$ \\
\hline C vs E & 4.7115 & 0.0115337 & $\mathrm{p}<0.05$ \\
\hline C vs F & 2.3695 & 0.5415625 & insignificant \\
\hline D vs E & 5.6247 & 0.0010053 & $\mathrm{p}<0.01$ \\
\hline D vs F & 12.7058 & 0.0010053 & $\mathrm{p}<0.01$ \\
\hline E vs F & 7.081 & 0.0010053 & $\mathrm{p}<0.01$ \\
\hline
\end{tabular}

\section{Discussion}

Changing the variety and molarity of the electrolyte present during the electrochemical exfoliation process has a definite effect on the electrical resistance of the films made using the process. However, the results are not entirely consistent, which raises many points of discussion and areas of future research. The $0.1 \mathrm{M}$ molarity sodium sulfate solution has a drastically different resistance level when compared to the $0.2 \mathrm{M}$ molarity solution, changing from the solution with the highest variance and resistances to the solution with the lowest. On the other hand, the 0.1M molarity potassium sulfate solution has a statistically insignificant change when the molarity is doubled. This is curious, since the chemicals are almost completely identical except for molar mass.

There are many possible explanations for why these two electrolytes exhibit such different behaviors, but there are three that are the most plausible. The first possibility is that the results were contaminated by pollutants. Although all of the electrochemical exfoliation reaction solutions were mixed with $99 \%+$ purity chemical sources, the potassium sulfate seemed to have by far the most impurities, with many small stones settling to the bottom. This quality was not shared by the other two, most likely due to different extraction methods. These impurities may have greatly changed the results of the reaction and caused the reaction's results to look similar despite an increased molarity.

The second possibility is that the potassium is not dissolving fully, which would limit its ability to exfoliate the graphite foil well. Sodium sulfate and especially ammonium sulfate are incredibly soluble in water when compared to potassium sulfate. Sodium sulfate is nearly twice as soluble in water, and ammonium sulfate is seven times as soluble. This lack of solubility may be affecting the reaction rate and making the addition of more solute ineffective. 
The third possibility is that potassium sulfate is exfoliating the foil too quickly, which causes damage to the flakes and therefore reduces the films' electrical resistance. Because the speed of the reaction seems tied to the voltage and the potassium sulfate solution has consistently completed the exfoliation process the most quickly, the flakes may be sustaining damage from the reaction that lowers their integrity and induces impurities like graphene oxide nodes on the films. This would in turn lower conductivity, but the damage sustained would not increase depending on concentration because the time taken for exfoliation is roughly similar.

When compared to other examples, this paper's measurements match well to the expected values for graphene's resistance. H. Wang et al. obtained a resistance of $1.21 \mathrm{k} \Omega$ when using sodium hydroxide as the electrolyte, which shows the potential of sulfate salts as a method of producing graphene. ${ }^{5}$ This is far superior to that of graphene produced using acidic electrolytes, which a different study led by $\mathrm{C}$. Chen et al. found to have a sheet resistance of $13.5 \mathrm{k} \Omega .{ }^{11}$ With an average resistance of $0.424 \mathrm{k} \Omega$, this study matches closely, and suggests that sulphate-based electrolytes may be highly efficient when exfoliating graphene. Further studies should be taken in order to prove consistent results, however.

\section{Limitations}

This study can be expanded on in a variety of areas, and many of them could lead to entirely new areas of research. The results from the data analysis could be used in many different ways, and many concepts explored here could be applied in different fields.

Although results were as ensured to be as consistent as possible with the given materials, there are still some areas where the study's accuracy could be improved. A multimeter is far from ideal when testing the resistance of a thin film like graphene due to its inaccuracy and potential for human error. In contrast, a four point probe system would be far more effective at finding incredibly accurate results because it uses 4 spring-mounted probes to carefully measure the top layer of the material without touching the bottom or scratching the surface. The study's budget made it impossible to afford such a system, but the use of one could lead to significant improvements.

Additionally, the purity of the electrolytes was questionable, as a 99\% purity leaves a surprisingly high amount of room for impurities. Using more pure electrolytes would improve the consistency and quality of the graphene films, although the price increases significantly for small improvements in purity.

Finally, the study was done in an environment that was reasonably sterile but conducting the experiment in a dedicated cleanroom would increase the film quality by reducing the amount of dust that settles on the films during the drying process. This would improve the quality of the films further, which would lead to more consistent and accurate results.

\section{Future Directions}

The data analysis results for the sodium sulfate and the potassium sulfate solution-produced graphene films are interesting and should be investigated further. Replication of this study and the addition of more advanced characterization techniques like Raman spectroscopy could quickly narrow down the cause of this discrepancy, and perhaps even lead into new insights about how the electrochemical exfoliation process works on a chemical level. Additionally, adding processes like chemical annealing and doping could further augment the success of graphene films, reducing resistance down far further than observed here.

The simplest addition to this study's field of research would be replicating it with additional electrolytes and observing the changes in conductivity. Attempting it with chemicals like sodium hydroxide, sulphuric acid, and lithium perchlorate might yield considerable results that could be investigated further. These chemicals are considerably more dangerous, however, and would require far more advanced facilities to safely use. 
Another direction that could be taken with this research is the actual application of graphene films, and how they hold up under continued use. Research has already been done concerning the use of graphene in batteries, and continued exploration into the application of small graphene films in electronics could provide immense dividends. Because this study outlines a method of producing graphene that is absolutely scalable, it could be increased to produce films on a massive scale. Easy access to films would also increase the ability for films to be experimented on, multiplying the research opportunities further. The possibilities that graphene films provide are immense and finding the most efficient method to produce them is paramount to the further development of next-generation electronics.

\section{Conclusion}

Especially when considering electronic applications, few-layer graphene and monolayer graphene have incredible potential. However, producing large amounts of usable graphene has been challenging due to the time and expenses required. This study aimed to evaluate the effect of varying sulfate electrolytes on the electrochemical exfoliation process in order to identify how the electrical resistance of graphene films was affected by different flake qualities. It found that there was a significant difference when changing the electrolyte's type and molarity, with the exception of changing the molarity of the potassium sulfate solution.

This knowledge can be applied in many practical fields of research, since graphene is one of the most commonly researched materials when concerning advanced electronics. If a specific resistance is needed, graphene made specifically with a certain resistance range could be fabricated to function as an atomic-scale resistor. If the exfoliation and film creation process is improved enough, graphene films could even become the stable method of electric transportation in electronics due to their extremely low resistance. The mass production of graphene is one of the most pursued areas when concerning nanotechnology, and the process of electrochemical exfoliation is a promising method of doing so.

\section{Acknowledgments}

I would like to thank my mentors Stefanie Davis and Mangilal Agarwal for helping me with this project.

\section{References}

Li D, Kaner RB. Graphene-based materials. Sciencemag. 2008; 320 (5880): 1170-1171. doi:10.1126/science. 1158180

Yan Z, Peng Z, Sun Z, et al. Growth of bilayer graphene on insulating substrates. ACS Nano. 2011; 5 (10): 8187 8192. doi:10.1021/nn202829y

Liu F, Wang C, Sui X, et al. Synthesis of graphene materials by electrochemical exfoliation: Recent progress and future potential. Carbon Energy. 2019; 1(2): 173-199. doi:10.1002/cey2.14

Dreyer DR, Ruoff RS, Bielawski CW. From conception to realization: an historical account of graphene and some perspectives for its future. Angewandte Chemie International Edition English. 2010; 49 (49): 9336-9344. doi:10.1002/anie.201003024

Wang H, Wei C, Zhu K, et al. Preparation of graphene sheets by electrochemical exfoliation of graphite in confined space and their application in transparent conductive films. ACS Applied Material Interfaces. 2017; 9 (39): 3445634466. doi:10.1021/acsami.7b09891 
Su C-Y, Lu A-Y, Xu Y, Chen F-R, Khlobystov AN, Li L-J. High-quality thin graphene films from fast electrochemical exfoliation. ACS Nano. 2011; 5 (3): 2332-2339. doi:10.1021/nn200025p

$\mathrm{Li} \mathrm{Z}$, Wu P, Wang C, et al. Low-temperature growth of graphene by chemical vapor deposition using solid and liquid carbon sources. ACS Nano. 2011; 5 (4): 3385-3390. doi:10.1021/nn200854p

Wei D, Grande L, Chundi V, et al. Graphene from electrochemical exfoliation and its direct applications in enhanced energy storage devices. Chem Commun. 2012; 48 (9): 1239-1241. doi:10.1039/C2CC16859F

Tripathi P, Patel CRP, Shaz MA, Srivastava ON. Synthesis of high-quality graphene through electrochemical exfoliation of graphite in alkaline electrolyte. arXiv:13107371. Published online October 28, 2013. Accessed March 5, 2021. http://arxiv.org/abs/1310.7371

Liu J, Poh CK, Zhan D, et al. Improved synthesis of graphene flakes from the multiple electrochemical exfoliation of graphite rod. Nano Energy. 2013; 2 (3): 377-386. doi:10.1016/j.nanoen.2012.11.003

Chen C-H, Yang S-W, Chuang M-C, Woon W-Y, Su C-Y. Towards the continuous production of high crystallinity graphene via electrochemical exfoliation with molecular in situ encapsulation. Nanoscale. 2015; 7 (37): 1536215373. doi:10.1039/C5NR03669K 